\title{
Effect of Continuous ATP Injection on Human Hemodynamics
}

\author{
Kimiaki Komukai, MD; Koichi Hashimoto, MD; Takahiro Shibata, MD; \\ Keiji Iwano, MD; Makoto Muto, MD; Junichi Mogi, MD; \\ Kamon Imai, MD*; Toshinobu Horie, MD*; Seibu Mochizuki, MD
}

\begin{abstract}
Continuous ATP injection is used clinically for Tl imaging or coronary flow measurement and because the effect on human hemodynamics is unknown, the present study investigated it in 14 patients undergoing heart catheter examination. Continuous ATP injection induced chest symptoms in 13 of the patients and second-degree atrioventricular block in one, but these complications disappeared immediately after the end of ATP infusion. Continuous ATP injection decreased aortic pressure, but increased pulmonary artery pressure, right atrial pressure and pulmonary capillary wedge pressure. ATP increased heart rate, stroke volume and cardiac output, the latter the result of an increase in preload, a decrease in afterload, and the increase in heart rate. (Circ J 2002; 66: 926-929)
\end{abstract}

Key Words: Adenosine triphosphate; Aortic pressure; Pulmonary artery

B ecause exogenous adenosine is known to induce hyperemia, it is used clinically for $\mathrm{Tl}$ imaging ${ }^{1}$ and coronary flow or pressure measurement? 23 In Japan, ATP is used for this purpose instead of adenosine $e^{4}$ because when administered intravenously, ATP is degraded to adenosine; therefore, the effects of adenosine and ATP are considered essentially the same. However, the direct effect of exogenous ATP might be manifiested through adenine nucleotide receptors ( $\mathrm{P}_{2 \mathrm{X}}, \mathrm{P}_{2} \mathrm{Y}$ ) in addition to its effect as adenosine through the adenosine receptors $\left(\mathrm{A}_{1}, \mathrm{~A}_{2}, \mathrm{~A}_{3}\right)$.

Adenosine $\mathrm{A}_{2}$ receptor stimulation relaxes smooth muscle via the stimulation of adenylyl cyclase activity. KATP channel opening via the A1 receptor stimulation may also relax vascular muscle. In cardiac muscle, $\mathrm{A}_{1}$ receptor stimulation has a negative inotropic effect: ${ }^{5}$ it decreases adenylyl cyclase activity, decreases L-type Ca current (ICa), shortens the action potential duration ${ }^{6}$ and inhibits contraction?

During continuous ATP injection, the direct effect of ATP should be considered in addition to the effect of its metabolite, adenosine. ATP induces vasodilation through the $\mathrm{P}_{2} \mathrm{Y}$ receptor, which is endothelium-dependent, and vasoconstriction through the $\mathrm{P}_{2} \mathrm{X}$ receptor. $\mathrm{P}_{2} \mathrm{Y}$ receptor stimulation inhibits cardiac muscle contraction through the inhibition of Ica?

Thus, there are many effects on cardiac and vascular smooth muscle from continuous ATP injection. Although the effect of adenosine on human hemodynamics is available from the literature, 2,8 the effect of continuous ATP injection is unknown and needs to be clarified.

(Received April 24, 2002; revised manuscript received July 1, 2002; accepted July 11, 2002)

Division of Cardiology, Department of Internal Medicine, The Jikei University School of Medicine, Tokyo and *Department of Cardiology, Saitama Cardiovascular and Respiratory Center, Konan, Japan Mailing address: Kimiaki Komukai, MD, Division of Cardiology, Department of Internal Medicine, The Jikei University School of Medicine, 3-25-8 Nishishinbashi, Minato-ku, Tokyo 105-8461, Japan. E-mail: komu@jikei.ac.jp

\section{Methods}

This study was performed in patients undergoing cardiac catheterization for re-evaluation after percutaneous coronary intervention $(\mathrm{PCI})$ or coronary aorta bypass graft surgery $(\mathrm{CABG})$ in Saitama Cardiovascular and Respiratory Center. Patients with acute coronary syndrome, severe heart valve disease, systemic hypotension, bronchial asthma, and any degree of atrioventricular (AV) block were excluded. Patients whose electrocardiogram (ECG) was not showing normal sinus rhythm or whose left ventricular (LV) ejection fraction was less than $40 \%$ were also excluded. Informed consent was obtained from each patient before the study.

First, diagnostic coronary angiography was performed using the Jadkins' technique. If significant stenosis existed in a major coronary artery that was not protected by a bypass graft, the patient was withdrawn from the study. The total number of patients was 14 (13 male; 1 female; post PCI, 7; post CABG, 7; mean age, $65.2 \pm 2.5$ years).

Right atrial pressure (RAP), right ventricular pressure (RVP) and pulmonary capillary wedge pressure (PCWP) were measured and the cardiac index (CI) and stroke volume index (SVI) were calculated using a thermodilution technique. The catheter was then mounted in the pulmonary artery (PA) and aorta (Ao) for simultaneous monitoring of PA pressure (PAP) and Ao pressure (AoP) while ATP was injected from a peripheral vein at $0.16 \mathrm{mg} \cdot \mathrm{kg}^{-1} \cdot \mathrm{min}^{-1}$. After 4 or 5 min of equilibration, PAP and AoP became steady-state, at which time PCWP, cardiac index (CI),

Table 1 Side Effects of Continuous ATP Injection

\begin{tabular}{lr}
\hline \hline & $n(\%)$ \\
\hline Chest symptoms & $13(93)$ \\
Pain & $10(71)$ \\
Discomfort & $3(21)$ \\
Headache & $3(21)$ \\
II AV block (Mobitz) & $1(7)$ \\
\hline
\end{tabular}



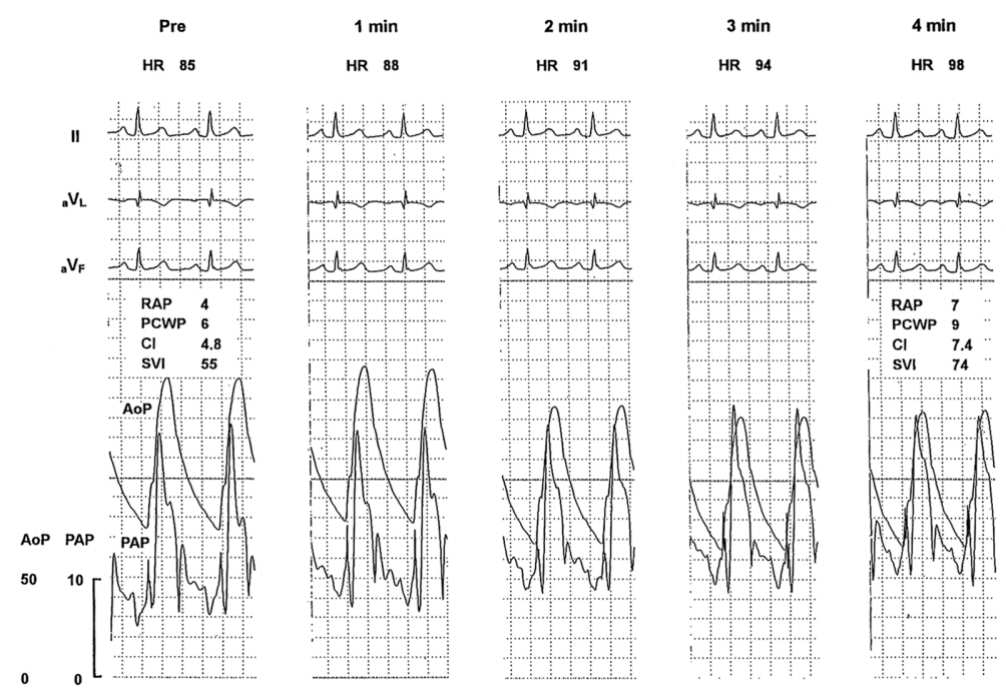

Fig 1. Original traces of ECG, aortic pressure (AoP) and pulmonary artery pressure (PAP) before and during ATP injection. HR, heart rate. RAP, mean right atrial pressure. PCWP, mean pulmonary capillary wedge pressure. CI, cardiac index. SVI, stroke volume index.

Table 2 Effect of Continuous ATP Injection on Hemodynamics

\begin{tabular}{lcccc}
\hline \hline & Control & ATP & $n$ & $p$ value \\
\hline HR $($ beats $/ \mathrm{min})$ & $73.5 \pm 3.4$ & $87.4 \pm 4.5$ & 14 & $p<0.001$ \\
Syst AoP $(\mathrm{mmHg})$ & $135 \pm 8$ & $120 \pm 8$ & 12 & $p<0.05$ \\
DP $\left(\mathrm{mmHg} \cdot \mathrm{beats} \cdot \mathrm{min}^{-1}\right)$ & $10,065 \pm 631$ & $10,725 \pm 834$ & 12 & $\mathrm{NS}$ \\
Mean AoP $(\mathrm{mmHg})$ & $98.7 \pm 5.7$ & $79.9 \pm 5.7$ & 13 & $p<0.05$ \\
Mean PAP $(\mathrm{mmHg})$ & $14.3 \pm 1.1$ & $15.7 \pm 1.2$ & 13 & $p<0.05$ \\
PCWP $(\mathrm{mmHg})$ & $7.9 \pm 1.1$ & $9.9 \pm 1.3$ & 13 & $p<0.01$ \\
RAP $(\mathrm{mmHg})$ & $4.0 \pm 0.5$ & $5.6 \pm 0.7$ & 14 & $p<0.01$ \\
CI $\left(\mathrm{ml} \cdot \mathrm{min}-1 . \mathrm{m}^{-2}\right)$ & $3.5 \pm 0.3$ & $5.0 \pm 0.5$ & 12 & $p<0.01$ \\
SVI $(\mathrm{ml} / \mathrm{min})$ & $43.4 \pm 2.4$ & $60.9 \pm 4.5$ & 12 & $p<0.001$ \\
SVR $\left(\right.$ dynes $\left.\cdot \mathrm{s} \cdot \mathrm{cm}^{-5} \cdot \mathrm{m}^{2}\right)$ & $2,739 \pm 333$ & $1,404 \pm 172$ & 11 & $p<0.001$ \\
TPR $\left(\right.$ dynes $\left.\cdot \mathrm{s} \cdot \mathrm{cm}^{-5} \cdot \mathrm{m}^{2}\right)$ & $349 \pm 41$ & $276 \pm 38$ & 12 & $p<0.001$ \\
PAR $\left(\right.$ dynes $\left.\cdot \mathrm{s} \cdot \mathrm{cm}^{-5} \cdot \mathrm{m}^{2}\right)$ & $161 \pm 19$ & $97 \pm 14$ & 12 & $p<0.001$ \\
\hline
\end{tabular}

HR, heart rate; Syst AoP, systolic aortic pressure; DP, double product; mean AoP, mean aortic pressure; mean PAP, mean pulmonary artery pressure; PCWP, mean pulmonary capillary wedge pressure; RAP, mean right atrial pressure; CI, cardiac index; SVI, stroke volume index; SVR, systemic vascular resistance; TPR, total pulmonary resistance; PAR, pulmonary arteriolar resistance.

stroke volume index (SVI), RVP and PAP were also measured.

Data are expressed as mean \pm SE for $n$ patients. Student's t-test for paired samples was performed and statistical significance was verified at $\mathrm{p}<0.05$ (two-tailed).

\section{Results}

During the ATP injection, minor complications appeared (Table 1): 13 of 14 patients had chest symptoms, which were transient in most cases or disappeared when the injection was stopped. One patient showed second-degree AV block (Mobitz II), which disappeared immediately after the end of the injection.

Fig 1 shows the original traces of ECG, AoP and PAP before and during ATP injection. ATP significantly increased heart rate (HR) but decreased systolic AoP; overall, double product (DP) was maintained. ATP significantly decreased mean AoP. On the other hand, ATP did not decrease but rather increased RAP, PAP and PCWP. ATP increased CI and SVI. Systemic vascular resistance (SVR), total pulmonary resistance (TPR), and pulmonary arteriolar resistance (PAR) were all decreased by ATP (Table 2).

\section{Discussion}

Side Effect of ATP Injection

ATP was injected at the dose routinely used for $\mathrm{Tl}$ imaging. Almost all patients $(13 / 14,93 \%)$ had chest symptoms. Mehta et al reported that adenosine-induced chest pain is not related to myocardial ischemia, but it is difficult to distinguish these symptoms from ischemia-induced symptoms outside the catheterization laboratory. In the present study, chest symptoms were not related to coronary artery stenosis, which was confirmed by coronary angiography.

Only 1 of the 14 patients showed second-degree AV block during ATP injection, but it disappeared immediately after the end of the injection. This effect is probably from adenosine-induced inhibition of the AV node. Adenosine opens muscarinic $\mathrm{K}^{+}$channels, which are concentrated in sinoatrial (SA) node and AV nodes, and so AV conduction might be inhibited. However, it is not sure why this inhibitory effect was not consistently observed in all patients. The dose used in the present study might not be enough to induce bradycardia in most of the patients.

Degradation of ATP and adenosine is very fast, so the serum concentration decreases very quickly and thus complications disappear immediately after the end of the injection. ATP can be used very safely. 
Table 3 Effect of Vasodilators on Hemodynamics

\begin{tabular}{|c|c|c|c|c|c|c|c|}
\hline & $H R$ & $R A P$ & $P C W P$ & $P A P$ & $A o P$ & $S V I$ & $C I$ \\
\hline Captopril $^{4}$ & $\downarrow$ & $\downarrow$ & $\downarrow$ & $\downarrow$ & $\downarrow$ & $\rightarrow$ & $\rightarrow$ \\
\hline Hydralazine ${ }^{11}$ & $\uparrow$ & $\rightarrow$ & $\rightarrow$ & $\downarrow$ & $\downarrow$ & $\uparrow$ & $\uparrow$ \\
\hline Nitroglycerin ${ }^{11}$ & $\rightarrow$ & $\downarrow$ & $\downarrow$ & $\downarrow$ & $\rightarrow$ & $\rightarrow$ & $\rightarrow$ \\
\hline Diltiazem $^{15}$ & $\downarrow$ & & $\rightarrow$ & & $\downarrow$ & $\uparrow$ & $\rightarrow$ \\
\hline Nifedipine ${ }^{10}$ & $\uparrow$ & $\rightarrow$ & $\rightarrow$ & $\rightarrow$ & $\downarrow$ & $\rightarrow$ & $\rightarrow$ \\
\hline Adenosine $e^{2,8}$ & $\uparrow$ & $\uparrow$ & $\uparrow$ & $\uparrow$ & $\downarrow$ & $\uparrow$ & $\uparrow$ \\
\hline ATP & $\uparrow$ & $\uparrow$ & $\uparrow$ & $\uparrow$ & $\downarrow$ & $\uparrow$ & $\uparrow$ \\
\hline
\end{tabular}

$H R$, heart rate; RAP, mean right atrial pressure; PCWP, mean pulmonary capillary wedge pressurel; PAP, mean pulmonary artery pressure; AoP, mean aortic pressure; SVI, stroke volume index; CI, cardiac index.

\section{Effect of ATP Injection onHR}

ATP and adenosine are considered to slow HR through the inhibition of both the SA and AV nodes. However, in the present study, ATP significantly increased HR, which is probably reflects the compensatory mechanism for its vasodilating effect. DP was maintained before and during the ATP injection. This increase in HR in response to a decrease in AoP is also observed when nifedipine ${ }^{10}$ or hydralazine ${ }^{11}$ is used (Table 3 ).

ATP or adenosine-induced stimulation of sympathetic nerves ${ }^{12}$ might also play a role in the increase in HR during ATP injection.

\section{Effect of ATP Injection on Vascular Muscle}

Intravenous injection of ATP induced a decrease in AoP and slight increase in PAP, RAP and PCWP. In the present study, ATP was injected from a peripheral vein, and its degradation occurred very quickly. It therefore appears possible that the concentrations of ATP and adenosine were different in the PA and Ao. The present results are qualitatively similar to those for adenosine, ${ }^{2,8}$ so the effect of ATP on PAP is likely to occur through adenosine. As for the PA, an increase in $\mathrm{CO}$ overcomes a decrease in vascular resistance, which results in an increase in PAP, while vascular resistance decreases enough to decrease pressure in the Ao. However, the mechanism of the different effect of ATP or adenosine on the PA and Ao is still unknown. Adenosine receptor density may be different in these vessels.

Fullerton et al reported that a low concentration of adenosine decreases PAP without changing AoP in patients with pulmonary hypertension ${ }^{13}$ In the present study, the basal PAP was within normal limits, so the effect of ATP or adenosine might differ between patients with pulmonary hypertension and those without it.

In contrast to captopril ${ }^{14}$ or nitroglycerin ${ }^{11}$ continuous ATP injection increased preload, observed as increases in RAP and PCWP. This increase in preload is also observed when adenosine is injected. According to Nussbacher et al, an increase in PCWP results from re-distribution of blood volume from the periphery to the central cortex?

\section{Effect of ATP Injection on Cardiac Function}

It is very difficult to evaluate contractility in humans because contractility should be independent of preload and afterload. But in the clinic, CI, SVI or the ejection fraction (EF) are used as a parameters for cardiac function, all of which are dependent on both preload and afterload.

In the present study, ATP did not decrease CI but rather increased it, partly because of an increase in HR. In isolated cardiac muscle, ATP or adenosine shows a negative inotropic effect; however, ATP significantly increased CI, which could be attributed to an increase in preload, a decrease in afterload, and an increase in HR.

\section{Comparison With Other Vasodilators (Table 3)}

Hydralazine and nifedipine increase HR in response to a decrease in AoP, as observed during ATP injection. Captropril and nitroglycerin decrease both preload and afterload, whereas hydralazine, nifedipine and diltiazem predominantly reduce afterload. A decrease in preload leads to a decrease in SVI, and a decrease in afterload leads to an increase in SVI; captopril and nitroglycerin do not alter SVI. Hydralazine, nifedipine, and diltiazem reduce afterload without affecting the preload, which favors to increase SVI. Hydralazine and diltiazem increase SVI but nifedipine fails to increase SVI, probably owing to a direct inhibitory effect on contractility. The effect of ATP is thus relatively similar to that of hydralazine.

Present results show that the effects of ATP and adenosine are essentially the same. The $\mathrm{P}_{2} \mathrm{X}$ and $\mathrm{P}_{2} \mathrm{Y}$ receptormediated effect might be small compared with the $\mathrm{A}_{1} / \mathrm{A}_{2}$ receptor-mediated effect. It also appears possible that the $\mathrm{P}_{2} \mathrm{X}$ and $\mathrm{P}_{2} \mathrm{Y}$ receptor-mediated effects are cancelled out by each other and masked by the $\mathrm{A}_{1} / \mathrm{A}_{2}$ receptor-mediated effect.

\section{References}

1. Iskandrian AE, Heo J. Myocardial perfusion imaging during adenosine-induced coronary hyperemia. Am J Cardiol 1997; 79: 20-24.

2. Ogilby JD, Ishandrian AS, Untereker WJ, Heo J, Nguyen TN, Mercuro J. Effect of intravenous adenosine infusion on myocardial perfusion and function. Hemodynamic/angiographic and scintigraphic study. Circulation 1992; 86: 887-895.

3. Ogliby JD. Role of adenosine in the cardiac catheterization laboratory. Am J Cardiol 1997; 79: 15-19.

4. Kobayashi Y, Yazawa T, Adachi T, Kawamura M, Ryu S, Asano T, et al. Ventricular arrhythmias with left bundle branch block pattern and inferior axis: Assessment of their mechanisms on the basis of response to ATP, nicorandil and verapamil. Jpn Circ J 2000; 64: $835-841$.

5. Komukai K, Kurihara S. Effects of adenosine on $\mathrm{Ca}^{2+}$ transient and tension in aequorin-injected ferret papillary muscles. Pflugers Arch 1994; 428: 357-363.

6. Nakai T, Watanabe I, Kunimoto S, Kojima T, Kondo K, Saito S, et al. Electrophysiological effect of adenosine triphosphate and adenosine on atrial and ventricular action potential duration in humans. Jpn Circ J 2000; 64: 430-435.

7. Qu Y, Himmel HM, Campbell DL, Strauss HC. Effects of extracellular ATP on ICa, $\left[\mathrm{Ca}^{2+}\right] \mathrm{i}$, and contraction in isolated ferret ventricular myocytes. Am J Physiol 1993; 264: C702-C708.

8. Nussbacher A, Arie S, Kalil R, Horta P, Feldman MD, Bellotti G, et al. Mechanism of adenosine-induced elevation of pulmonary capillary wedge pressure in humans. Circulation 1995; 92: 371-379.

9. Mehta AB, Mardikar HM, Hiregoudar NS, Sethi RB, Solanki DR, Mathew R. Adenosine-induced chest pain: Is it due to myocardial ischaemia? Clinical, electrocardiographic, haemodynamic and metabolic study. Ind Heart J 1997; 49: 267-270.

10. Burger W, Herholz H, Burger K, Kober G. Antiischemic and hemodynamic effects of intravenous isradipine, a new calcium antagonist, 
in coronary heart disease: A comparative double-blind cross-over study with nifedipine. J Cardiovasc Pharmacol 1990; 16: 764-768.

11. Roth A, Shotan A, Elkayam U. A randomized comparison between the hemodynamic effects of hydralazine and nitroglycerin alone and in combination at rest and during isometric exercise in patients with chronic mitral regurgitation. Am Heart J 1993; 125: 155-163.

12. Rongen GA, Senn BL, Ando S, Notarius CF, Stone JA, Floras JS. Comparison of hemodynamic and sympathoneural responses to adenosine and lower body negative pressure in man. Can J Physiol Pharmacol 1997; 75: 128-134.
13. Fullerton DA, Jones SD, Grover FL, McIntyre RC Jr. Adenosine effectively controls pulmonary hypertension after cardiac operations. Ann Thorac Surg 1996; 61: 1118-1124.

14. Massie B, Kramer BL, Topic N, Henderson SG. Hemodynamic and radionuclide effects of acute captopril therapy for heart failure: Changes in left and right ventricular volumes and function at rest and during exercise. Circulation 1982; 65: 1374-1381.

15. Ogawa H, Yasue H, Nakamura N, Obata K, Sonoda R. Hemodynamic effects of intravenous diltiazem in patients with acute myocardial infarction. Clin Cardiol 1987; 10: 323-328. 